\title{
ERRATUM
}

\section{Erratum to: Methodology for commissioning a brachytherapy treatment planning system in the era of 3D planning}

\author{
Claire Dempsey
}

Published online: 28 December 2010

(C) Australasian College of Physical Scientists and Engineers in Medicine 2010

\section{Erratum to: Australas Phys Eng Sci Med}

\section{DOI 10.1007/s13246-010-0036-2}

A calculation error has occurred in Table 3 of the original article published in OnlineFirst. The corrected Table 3 is shown below.

Table 3 Conformity Index (COIN) values calculated for three different treatment sites

\begin{tabular}{|c|c|c|c|c|c|}
\hline Treatment site & Plan & $\mathrm{CTV}\left(\mathrm{cm}^{3}\right)$ & $\operatorname{CTV}_{(100)}\left(\mathrm{cm}^{3}\right)$ & $V_{(100)}\left(\mathrm{cm}^{3}\right)$ & COIN \\
\hline \multirow[t]{2}{*}{ Prostate } & Non-optimized & 44.96 & 36.73 & 45.28 & 0.66 \\
\hline & IPSA & 44.96 & 44.11 & 64.37 & 0.67 \\
\hline \multirow[t]{2}{*}{ Vaginal Wall } & Non-optimized & 26.91 & 17.66 & 27.69 & 0.42 \\
\hline & IPSA & 26.91 & 19.71 & 29.57 & 0.48 \\
\hline \multirow[t]{2}{*}{ Uterine Cervix } & Non-optimized & 68.09 & 62.26 & 99.36 & 0.70 \\
\hline & IPSA & 68.09 & 67.85 & 96.39 & 0.57 \\
\hline
\end{tabular}

Each site had non-optimized and IPSA optimized plans created. The PTV was used as the CTV used in the COIN formula [21, 22]. The reference isodose was the $100 \%$ isodose line

The online version of the original article can be found under doi:10.1007/s13246-010-0036-2.

C. Dempsey $(\bowtie)$

Department of Radiation Oncology, Calvary Mater Newcastle

Hospital, Locked Bag 7, Hunter Regional Mail Centre,

Newcastle, NSW 2310, Australia

e-mail: Claire.hood@mater.health.nsw.gov.au

C. Dempsey

School of Health Sciences, University of Newcastle,

Newcastle, NSW, Australia 